\title{
In situ investigation by X-ray tomography of the overall and local microstructural changes occurring during partial remelting of an $\mathrm{Al}-15.8 \mathrm{wt} . \% \mathrm{Cu}$ alloy
}

\author{
Nathalie Limodin ${ }^{\mathrm{a}, *}$, Luc Salvo ${ }^{\mathrm{a}}$, Michel Suéry ${ }^{\mathrm{a}}$, Marco DiMichiel ${ }^{\mathrm{b}}$ \\ ${ }^{a}$ Institut National Polytechnique de Grenoble, SIMAP/GPM2, UMR CNRS 5266, UJF, BP 46, 38402 Saint-Martin d'Hères Cedex, France \\ ${ }^{\mathrm{b}}$ ESRF, 156 Rue des Martyrs, BP 220, 38043 Grenoble Cedex 9, France
}

The paper is concerned with the study of the microstructural changes occurring during holding of an $\mathrm{Al}-15.8 \mathrm{wt} . \% \mathrm{Cu}$ alloy in the semisolid state. These changes are investigated in 3D by in situ X-ray tomography carried out at the temperature of the treatment. The studies are classified in two categories: overall changes by measuring average values of characteristic parameters, and local changes by considering the evolution of individual necks between particles. It is shown in particular that the size of the solid particles or the surface area of the solid-liquid interfaces do not follow the classical power laws but rather evolve in a slower manner. Local observations confirm that these results are due to the competition of two coarsening mechanisms of the solid particles that occur simultaneously: dissolution of a small particle to the benefit of one or several bigger ones by an Ostwald-type mechanism and the growth of necks between solid particles due to their coalescence. Complex variations of neck size result from these mechanisms which can be explained only by considering the neighbourhood of the particles under investigation. These observations confirm that in situ X-ray tomography is a very powerful tool to provide data that are representative of the semi-solid state and to observe in real time the mechanisms that act on the microstructure.

Keywords: Semi-solid processing; 3D X-ray tomography; Aluminium alloys; Equiaxed microstructure; Coarsening

\section{Introduction}

The evolution of the microstructure of aluminium alloys during partial remelting and holding in the semi-solid state has been widely investigated in recent years owing to the development of semi-solid processing for forming applications. The main objective is to produce a fine globular structure which exhibits thixotropic properties. The starting microstructure can be either dendritic or rosette-like or even globular, depending on the initial treatment applied to the alloy.

In the case of $\mathrm{Al}-\mathrm{Cu}$ alloys, the evolution of the microstructure during isothermal holding in the semi-solid state

\footnotetext{
* Corresponding author. Tel.: +334768271 60; fax: +33476826382. E-mail address: nathalie.limodin@gpm2.inpg.fr (N. Limodin).
}

has been studied, starting from columnar dendritic microstructures [1-3]. The evolution of the dendritic morphology is then usually followed through $S_{\mathrm{v}}$, the area of the solidliquid interface normalized by the total volume, or through the average secondary dendrite arm spacing. In such cases, the chosen characteristic size was reported to vary as $t^{-1 / n}$, where $n$ can take different values. Most studies found a value of $3[4]$ but some [1,5] observed smaller $n$ values with sometimes two stages in the time evolution of $S_{\mathrm{v}}$ characterized by different $n$ values and different "coarsening" mechanisms for each stage.

Numerous theories were proposed to predict the evolution of microstructural parameters during holding in the semi-solid state. Kattamis $[1,2,6]$ developed several models that can be numerically integrated. They describe the evolution of dendritic microstructures quite well and they lead 
to various $n$ values ranging from 1 to 9 depending on the alloy composition, the time range and the starting microstructure [1]. Mortensen [7] also developed a model that predicts a time evolution with $n=3$. The one-third coefficient is usually associated with Ostwald ripening in structures with a low solid volume fraction and a high grain boundary energy; it is mostly observed at long times when asymptotical behaviour is reached though it has been also reported at short holding time in [5].

Studies have been performed also on initially globular or equiaxed dendritic microstructures [5,8]. In some cases (at volume fractions of the coarsening phase greater than 0.5 ), exponents smaller than one-third were observed $[5,9,10]$. Even at long holding times when dendritic structures have become globular, the exponent remains very small and depends on the coarseness of the initial microstructure [5]. This is ascribed to the predominance of a coalescence mechanism that is made possible owing to a ratio of the solid-solid to the solid-liquid interfacial tension less than 2 in $\mathrm{Al}-\mathrm{Cu}$ alloys.

Many models predict the coalescence of spherical particles in liquid phase sintering. Some of them such as Courtney's model [11-13] can be adapted to semi-solid alloys. Courtney assumed that densification is complete and that the kinetics of coalescence is controlled by volume diffusion of solute in the liquid phase; these conditions are similar to those prevailing in the semi-solid state. Courtney established "maps" that predict which coarsening mechanism is dominant depending on the microstructure (isolated spheres or skeletal microstructure), on the solid volume fraction, and on a parameter that measures the relative rate of ripening compared to coalescence $[12,13]$. For short holding times when coalescence is predominant, Courtney $[11,13]$ predicts a time evolution of the surface area of the solid different from the classical Lifshitz-Slyozov-Wagner (LSW) theory for Ostwald ripening. In particular, his model is based on a smaller $1 / n$ exponent, i.e. from $1 / 6$ to $1 / 5$ depending on the time range, though it is not as small as the exponents found by Poirier at long holding times. There is some controversy about the time range for which Ostwald ripening or coalescence is predominant. Courtney assumes that coalescence occurs first whereas, according to Bender and Ratke [14], coalescence requires that particles grow by ripening to make new coalescence contacts so that it can occur at long holding times only. Indeed, Poirier et al. [5] observe ripening at short times, i.e. $S_{\mathrm{v}}$ follows a one-third power law with time, and coalescence at long holding times, i.e. $S_{\mathrm{v}}$ follows a power law with a small coefficient. Therefore, studies based on the real-time observation of the microstructure would help to clarify this point.

None of the above-mentioned models takes into account the influence of the neighbourhood or the size distribution of the solid particles. LSW predicts that a particle will grow or shrink at a rate that depends on its relative size as compared to the average [15]. Courtney assumes that coalescence and ripening occur separately at different time ranges, and thus he ignores the possible interactions between mechanisms.

Experimental measurements in the above-mentioned papers were carried out on 2D metallographic sections obtained after quenching the specimen from the semi-solid state. Two-dimensional data give information on the specific surface value but they give very little insight into the evolution of local curvatures of the solid-liquid interface although this would allow a better following and understanding of the operative coarsening mechanisms. Thus, three-dimensional information is required. Voorhees and co-authors [16] used serial sectioning to follow the morphological evolution of a dendritic $\mathrm{Al}-\mathrm{Cu}$ alloy and to show the corresponding evolution of local Gaussian and mean curvatures for two isothermal holding times. With the same technique, they were able to observe the distribution of particle-particle contacts in semi-solid $\mathrm{Sn}-\mathrm{Pb}$ alloys at different solid fractions [17], which would have been impossible with 2D data. Serial sectioning is a destructive technique that provides a $4.75 \mu \mathrm{m}$ spatial resolution and requires quenching from the mushy state: this might be a limitation since structural parameters depend strongly on the cooling rate and only very fast cooling or quenching from a temperature close to the eutectic temperature is able to give a microstructure that is representative of the semisolid state [18].

Microtomography offers another possibility to retrieve 3D information; because it is non-destructive, it allows following the evolution of local microstructural features inside a given specimen. Microtomography was first performed ex situ at room temperature: the specimen was quenched from the isothermal holding temperature to acquire the tomography scans. Some data were obtained with this technique and the interface surface area appeared to follow a one-third power-law variation with time [19]. Recently, the development of fast tomography allowed in situ scans to be made at every minute of isothermal holding so that the various parameters have been measured while the specimen is held in the semi-solid state. Significant differences were then detected between measurements of the globule size or the solid fraction of a quenched sample and a semi-solid sample [20].

The aim of this paper is to present a detailed experimental study of the microstructural evolution of an aluminium-copper alloy in the semi-solid state using in situ microtomography. Microstructural evolution will be characterized by the variation with time of standard average parameters but also by local observations concerning the operating mechanisms.

\section{Experimental methods}

\subsection{Material and X-ray microtomography device}

The Al-15.8 wt.\% $\mathrm{Cu}$ alloy was selected because the solid and liquid phases present good absorption contrast as soon as they have different compositions. Eutectic and 
liquidus temperatures of the alloy are 548.2 and $616.8^{\circ} \mathrm{C}$, respectively. Experiments were performed on a cylindrical specimen $1.5 \mathrm{~mm}$ in diameter and $3 \mathrm{~mm}$ in height. The specimen was first solidified in a metallic mould to obtain a fine starting microstructure. Then, it was glued with zirconia paste on the top of an alumina rod placed on the rotating stage. A small furnace developed at ESRF Grenoble (European Synchrotron Radiation Facility) was used to perform the thermal treatment as detailed in Ref. [21]. The sample was supported by its own oxide skin up to the temperature of the experiment. The specimen was held at a temperature of about $555^{\circ} \mathrm{C}$ during $80 \mathrm{~min}$ while X-rays were switched on and microtomography was carried out.

The experiments were realized on the ID15 beam line at the ESRF. X-rays go through the sample and a high-speed camera records the transmitted intensity while the sample is continuously rotating over $180^{\circ}$. Four hundred projections were taken over this $180^{\circ}$ rotation. Dark-field images, i.e. without X-rays, and flat field images, i.e. with X-rays but without the sample, were also recorded for further processing of the images. The readout time of the camera is less than $16.7 \mathrm{~ms}$ in full frame mode $(1024 \times 1024$ pixels $)$ and is reduced to $9.1 \mathrm{~ms}$ in binning mode $(512 \times 512$ pixels) . To decrease the scan time, the CCD region of interest was reduced to $512 \times 512$ pixels. The scan time over a total rotation is smaller than $15 \mathrm{~s}$, which is assumed to be less than the time required for significant microstructural changes. The final spatial resolution is $2.8 \mu \mathrm{m}$. A scan was taken every minute from $1 \mathrm{~min}$ to $80 \mathrm{~min}$ holding to characterize the evolution with holding time of the semisolid microstructure at a constant temperature.

\subsection{Image processing}

The reconstruction of the volumes from the projections has been detailed elsewhere [21]. After reconstruction, the images (Fig. 1a) are cropped to a final size of $263 \times 228 \times 250$ pixels, i.e. $0.7 \times 0.6 \times 0.7 \mathrm{~mm}$ in order to be able to perform $3 \mathrm{D}$ calculations with conventional PC computers. The image processing follows the steps indicated below:

1. First, a 3D median filter was applied to reduce the noise.

2 . In the greyscale image, a $3 \mathrm{D}$ region-growing algorithm was used to segment the solid and liquid phases.

3. The generated surface, obtained with a conventional marching cube algorithm using Amira software, was then smoothed. This leads to a volume that will be called $V_{\text {init }}$ (Fig. 1b).

4. Using volume $V_{\text {init }}$, solid particles were 3D segmented at each detectable neck by using an Aphelion built-in routine, i.e. "split convex". The routine automatically computes a 3D distance function (minimum distance to the liquid-solid interface to create a basin centred on the particle), then it applies a filter, and finally it separates the particles with a $3 \mathrm{D}$ watershed method leading to volume $V_{\text {solid }}$ (Fig. 1c). a

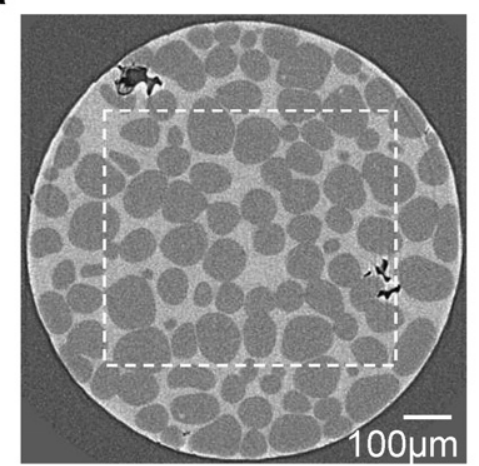

C

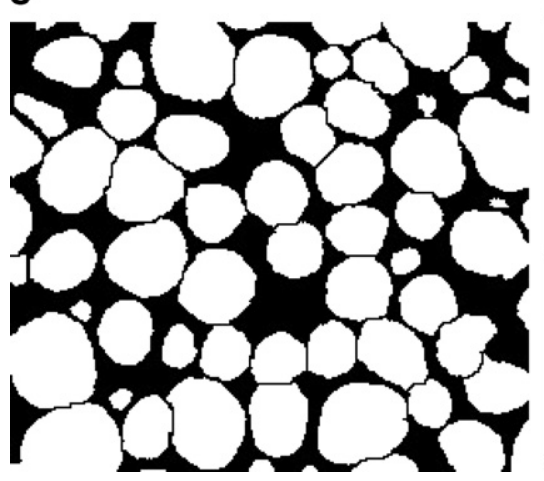

b

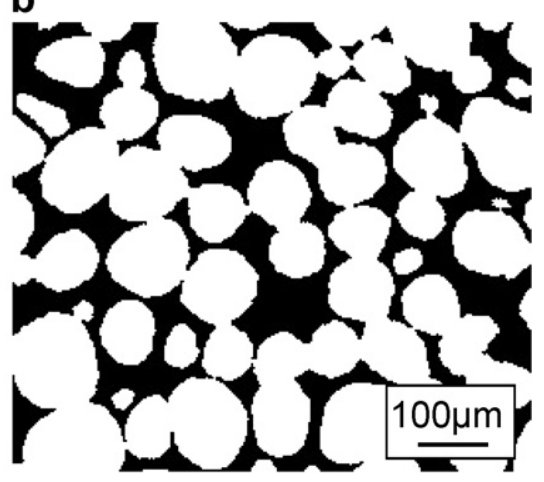

d

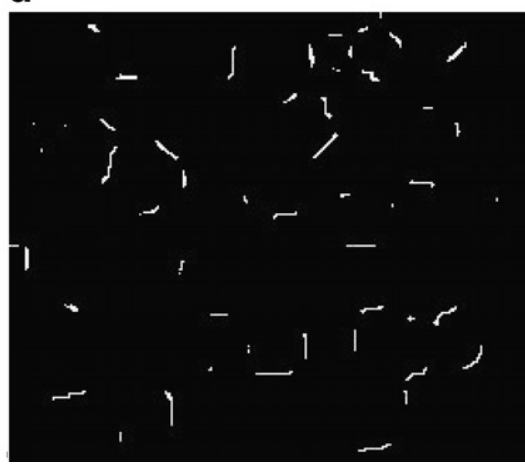

Fig. 1. Examples of 3D image processing with 2D slice at a given height in the cylindrical specimen: (a) grey-scale original image (solid is in dark grey), (b) solid (in white) and liquid (in black) in $V_{\text {init }}$, (c) segmented solid particles in $V_{\text {solid, }}$, and (d) isolated necks in $V_{\text {neck. }}$ 
5. A new volume, $V_{\text {neck }}$ (Fig. 1d), containing only the necks between the solid particles, was created by subtracting $V_{\text {solid }}$ from $V_{\text {init }}$.

6. From these three volumes, quantitative information is obtained not only at a global scale but also at a local scale:

- From $V_{\text {init }}$ the solid volume fraction and the solidliquid interface area are obtained.

- From $V_{\text {solid }}$ we obtained all the information on the solid particles (those touching the border are removed) such as the size distribution, the mean size, the shape factor defined as $s=6 \times V \times \sqrt{\pi / S^{3}}$ where $S$ is the surface and $V$ the volume, the coordination number, and the gravity centre.

- From $V_{\text {neck }}$ we obtained information on the size and gravity centre of the necks.

7. Using $V_{\text {solid }}$ or $V_{\text {neck }}$ at various times during the isothermal holding treatment, we were able to follow a specific solid particle or a specific neck (following their gravity centre) and thus to retrieve local microstructural features.

\section{Results}

\subsection{Preliminary results}

The first value of the solid volume fraction $g_{\mathrm{s}}$ (Fig. 2) that can be measured at the beginning of the isothermal holding, i.e. at $180 \mathrm{~s}$, is 0.66 . Afterwards, it increases until $1380 \mathrm{~s}$ and it progressively stabilizes at 0.68 after $3000 \mathrm{~s}$. A solid mass fraction $f_{\mathrm{s}}$ was calculated from $g_{\mathrm{s}}$ using the densities for the solid and liquid phases reported in Ref. [22]. The values so obtained are 0.63 and 0.65 for the initial and stable values of $f_{\mathrm{s}}$, respectively. These values are higher than the 0.55 value calculated from Scheil's

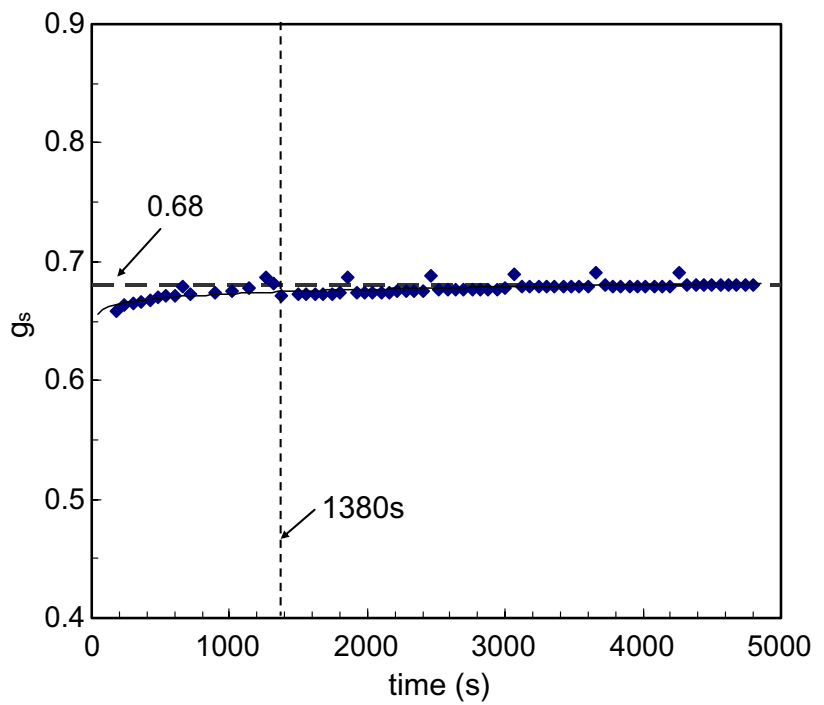

Fig. 2. Variation of the solid volume fraction with isothermal holding time. equation and the 0.59 value calculated at equilibrium assuming a temperature of $555^{\circ} \mathrm{C}$. This could result from a lack of temperature accuracy from the microtomography device, from uncertainty in the image threshold, or from inaccuracy in the densities or in the alloy composition. Nevertheless, the initial increase of the solid fraction up to $1380 \mathrm{~s}$ shows the trend expected for a progressive return of the specimen from non-equilibrium, with a mass solid fraction given by Scheil's equation, to thermodynamic equilibrium with diffusion of $\mathrm{Cu}$ in the solid phase. Progressive disappearing of liquid inclusions entrapped in the solid globules could also participate in the decrease of the liquid fraction [23] and in the corresponding increase of the solid fraction.

The evolution of the semi-solid microstructure during isothermal holding was characterized by monitoring some microstructural parameters as a function of time. Analysis was limited to holding times longer than about 1380 s, i.e. the time required for $g_{\mathrm{s}}$ to level off. Results of this investigation are reported below. The number of solid particles was counted after segmentation of the volume of size $0.7 \times 0.6 \times 0.7 \mathrm{~mm}$ without counting particles cut by the borders. Thus, average values of the parameters that describe the microstructure were measured for a solid globules population ranging from 244 at $1380 \mathrm{~s}$ to 189 after $4800 \mathrm{~s}$ of holding at $555^{\circ} \mathrm{C}$. This relatively low number of particles is at the moment the maximum that can be obtained in in situ conditions for this alloy with a $2.8 \mu \mathrm{m}$ spatial resolution. We believe it is large enough for identifying local mechanisms.

\subsection{Average values of microstructural parameters and distributions}

We investigate here several conventional parameters: the number of particles, the shape factor of the solid phase, the coordination number, the average size of the solid phase, the solid-liquid interface area and finally the mean neck size between the solid particles.

\subsubsection{Number of particles}

The number of particles in the analysed volume decreases with holding time. According to the LSW theory, the particle density $N_{\mathrm{v}}$ should be inversely proportional to time [24]. Courtney found a similar dependence for coalescence at sufficiently long holding times [13]. The fit that could be obtained assuming such a linear relationship is plotted in Fig. 3. Obviously, a better fit would be obtained with a different time dependency.

\subsubsection{Shape factor of solid particles}

According to the relationship presented above, the value of the shape factor is one for a perfect sphere and less than one for a non-spherical object. At $900 \mathrm{~s}$, the local shape factor ranges from less than 0.5 to 0.86 with an average value around 0.76 . With increasing holding time, the distribution is shifted towards higher values of the average shape 


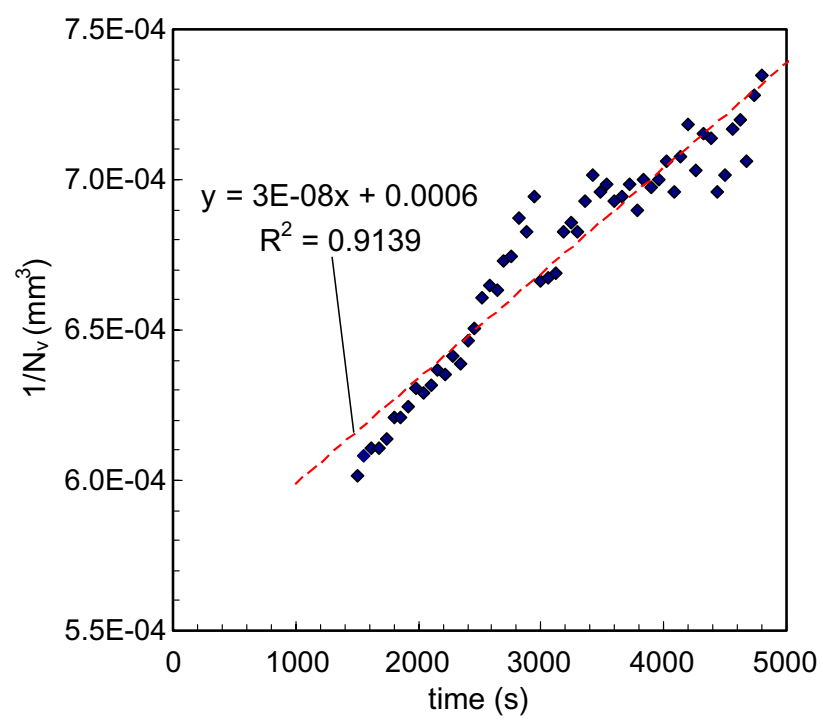

Fig. 3. Variation of the particle density with isothermal holding time.

factor while the peak width decreases. Even at long holding times, the maximum shape factor does not bypass 0.92 , i.e. there is no spherical particle. The curve in Fig. 4 shows that, at very short holding times $(t=180 \mathrm{~s})$, solid particles have an initial non-spherical shape $(s \approx 0.66)$ inherited from the dendritic microstructure that rapidly evolves towards a more globular shape $(s \approx 0.82)$ at $1500 \mathrm{~s}$. After $1500 \mathrm{~s}$, the spheroidization slows down and the average shape factor tends slowly to 0.845 . Shape evolution is illustrated by 3D rendering of a given globule between $1260 \mathrm{~s}$ and $2760 \mathrm{~s}$ in Fig. 4.

\subsubsection{Coordination number}

There is no free particle. A particle makes at least one contact and it can have a maximum of 13 neighbours. This

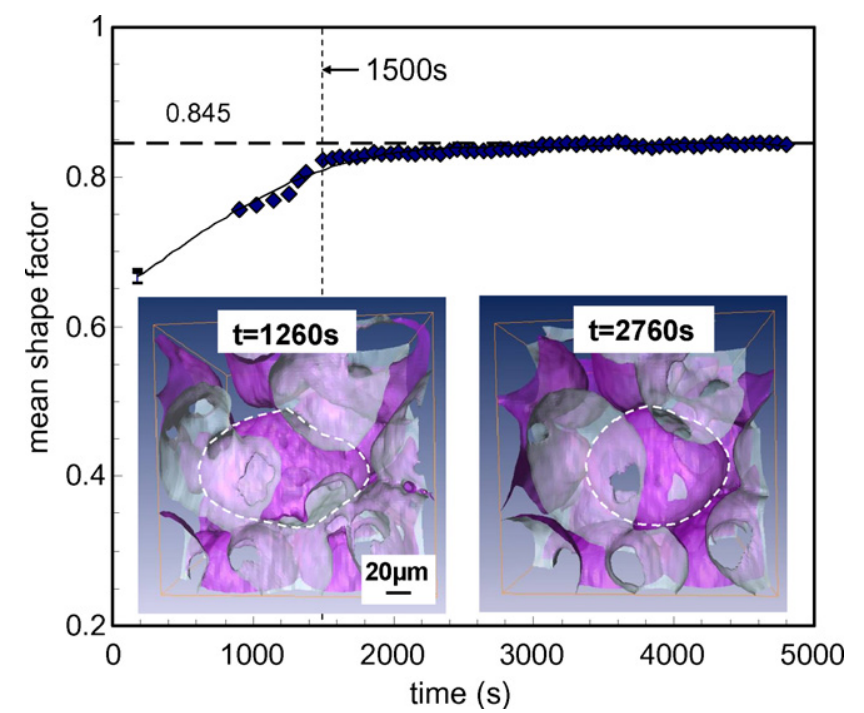

Fig. 4. Variation of the mean shape factor of the solid particles with isothermal holding time and illustrations of the local shape factor increase of a given particle from 0.71 at $1260 \mathrm{~s}$ to 0.87 at $2760 \mathrm{~s}$. can explain why a perfect spherical shape cannot be obtained; solid globules are connected by necks and cannot be considered as isolated spheres. The coordination number of a solid particle, $z$ in Fig. 5, decreases slightly from 6.5 to 6.2 from $1380 \mathrm{~s}$ to $3000 \mathrm{~s}$ before it appears to level off. These values are in agreement with results obtained by Rowenhorst et al. for the same kind of particle morphology [17].

\subsubsection{Size of solid particles}

From about $1500 \mathrm{~s}$ to the end of the isothermal treatment at $4800 \mathrm{~s}$, the mean shape factor of the solid particles is rather stable and close to that of a sphere (Fig. 4); therefore, the particle size can be characterized by an equivalent radius, which is the radius of a sphere having the same volume as the particle. At a holding time of $1500 \mathrm{~s}$, solid globules have equivalent radii spanning from $16 \mu \mathrm{m}$ to $81 \mu \mathrm{m}$ with an average value of $48.8 \mu \mathrm{m}$; this size range is retained with increasing holding time while the average value increases slowly (Fig. 6). The size distribution of the particles which is plotted in Fig. 7 is not perfectly self-similar with time when it is scaled by the average equivalent radius. Frequencies were normalized by the area of the distribution to be comparable with the distributions computed from the LSW [25] model and the Takajo et al. [26] model for coalescence. None was able to predict the exact distribution in our sample; the experimental distribution is not as large as the Takajo's distribution, in which two coalesced particles are considered to form one larger particle, and it spreads towards larger values of the relative equivalent radius than the LSW's distribution does. Fig. 6 shows that the average volume of the solid particles increases with time. This coarsening of the microstructure does not follow a continuous linear law except if we divide the timescale in two parts, i.e. from $1500 \mathrm{~s}$ to $3000 \mathrm{~s}$ then from $3000 \mathrm{~s}$ to

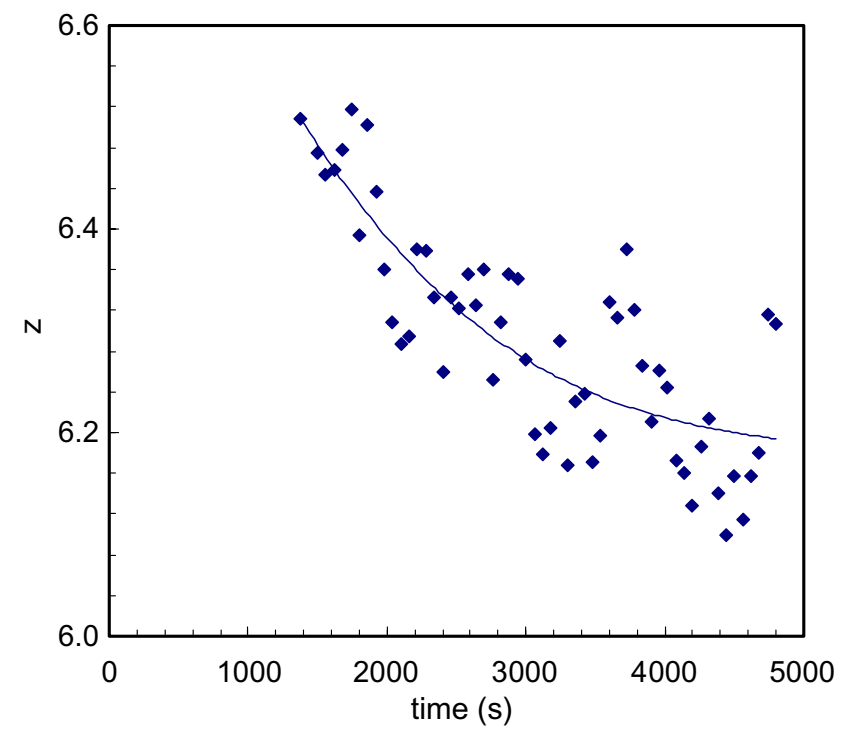

Fig. 5. Variation of the average coordination number with isothermal holding time. 


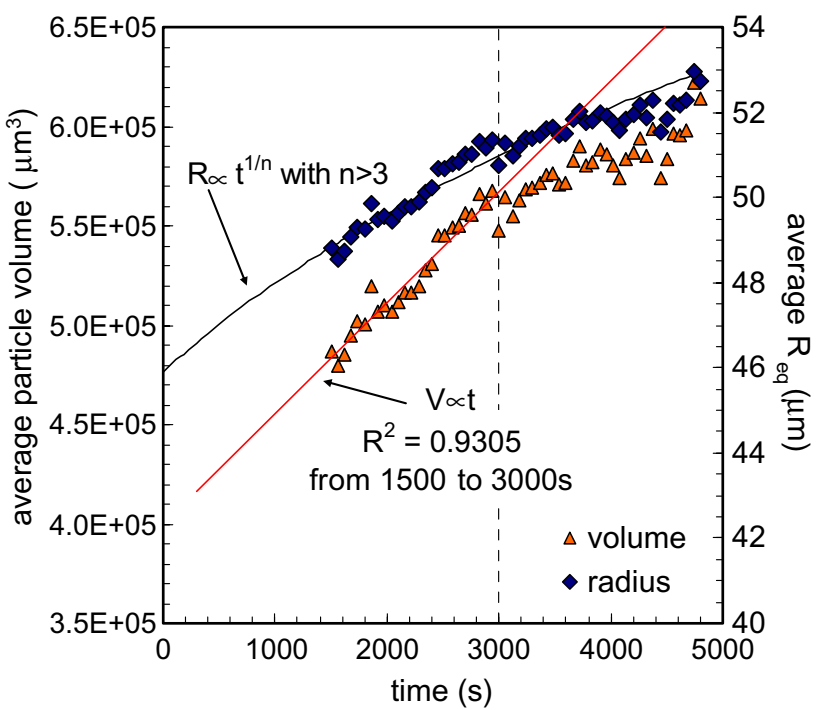

Fig. 6. Variation of the average solid particle volume and equivalent radius with isothermal holding time.

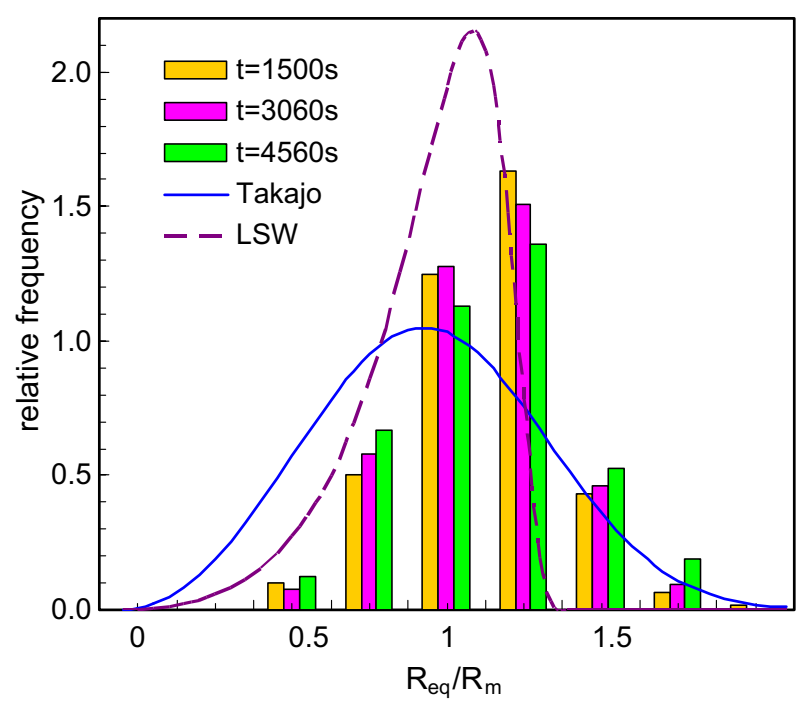

Fig. 7. Relative size distribution for different holding times and comparison with analytical curves from the literature.

$4800 \mathrm{~s}$. Consequently, it is not possible to fit the global evolution of the equivalent radius with the standard $t^{1 / 3}$ power law. Actually, a smaller exponent is found.

\subsubsection{Area of the solid-liquid interface}

$S_{\mathrm{v}}$ plotted in Fig. 8 is the surface area of the solid-liquid interface normalized by the total volume of analysis. It varies in inverse order of the average equivalent radius of the solid particles and it better characterizes the size of the microstructure than the equivalent radius because it does not make any assumption on the particle shape. The time evolution of $S_{\mathrm{v}}$ can be fitted with:

$S_{\mathrm{v}}=\left(0.061^{-n}+K \times t\right)^{-1 / n}$

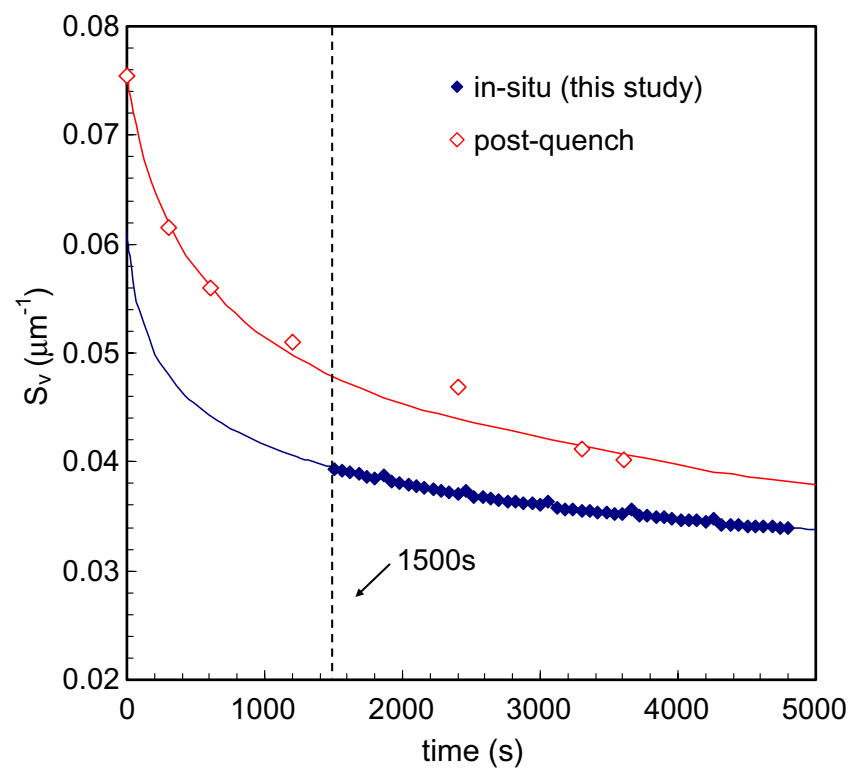

Fig. 8. Variation of $S_{\mathrm{v}}$ with time and comparison with post-quench measurements on the same alloy; the curve passing through the in situ experimental points corresponds to Eq. (1).

where $K=2.3 \times 10^{7} \mu \mathrm{m}^{n} \mathrm{~s}^{-1} \pm 0.7 \times 10^{7} ; n=7.5 \pm 0.09$.

This confirms that coarsening in the present alloy has a time dependency different from what is expected from the LSW theory for ripening of spheres dispersed in a liquid matrix. Poirier et al. [5] also observed $n$ exponents larger than three, i.e. from five to even higher values at long holding times for a similar material.

In Fig. 8, results of the post-quench measurements obtained in [19] were plotted for comparison.

\subsubsection{Neck size evolution}

The LSW model was developed for dispersed spheres assuming that the solid fraction tends to zero. In the present study, the solid fraction is rather high and solid globules are all connected by necks. Fig. 9 reports the variation with time of the average surface area of the necks; it can be fitted with a power law:

$S_{N m}=\left(275^{n}+K \times t\right)^{\frac{1}{n}}$

where $K=2.7 \times 10^{6} \mu \mathrm{m}^{2 n} \mathrm{~s}^{-1} \pm 1.6 \times 10^{6} ; n=2.9 \pm 0.08$.

Assuming a disk shape for the necks, Eq. (2) implies that the average neck radius is growing with time according to the power $1 / 5.8$.

The decrease of the number of the solid particles, the coarsening of the microstructure and the increase in the average neck size can correspond to the growth of the necks between coalescing particles as well as to the dissolution of small necks between particles of different sizes with one particle absorbing the smaller one. Analysis of average values does not allow distinguishing between the two coarsening mechanisms. Only the analysis of the evolution of 


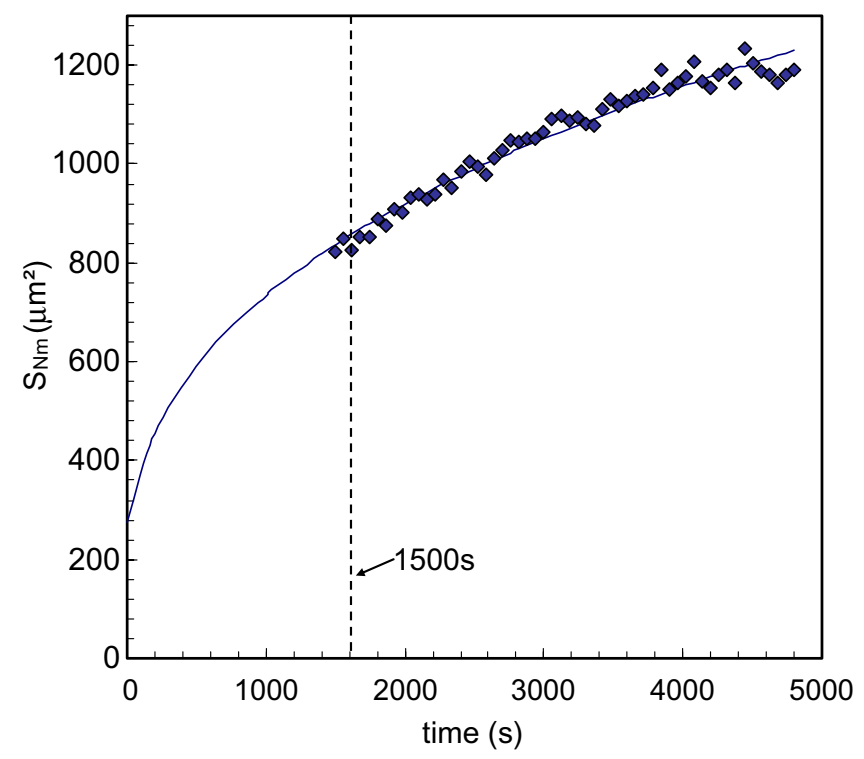

Fig. 9. Variation of the average surface of the necks between adjacent particles with isothermal holding time; the curve corresponds to Eq. (2).

local features could tell us which mechanisms are indeed playing a significant role on the microstructure evolution.

\subsection{Local evolution of the microstructure}

In order to identify the mechanisms that result in the increase of the average neck size, the evolution of 266 necks was followed from 1500 to $4800 \mathrm{~s}$ of isothermal holding. Table 1 presents the trends that were observed for the evolution of neck surface with time and their importance. For each trend, the neck size was normalized by $S_{N 0}$, the initial size of the neck at the time $t_{0}$ when it was detected, and reported as a function of the relative time $\left(t-t_{0}\right)$. Then, necks with an evolution representative of each trend (Fig. 10) were chosen for observation in 3D images with Amira software. For the sake of visibility, only the neck, the two particles that it joins, and some representative neighbours were drawn. Several trends have been observed with increasing holding time: indeed, the neck size

- decreases,

- increases,

Table 1

Identification of the various trends in necks evolution and determination of their respective weights during the period of time of the analysis

\begin{tabular}{lccl}
\hline Trend of necks evolution & Number & $\%$ & No. in Fig. 10 \\
\hline Decrease & 70 & 26.3 & $(1)$ \\
Increase & 69 & 25.9 & $(2)$ \\
Increase and decrease & 40 & 15.0 & $(6)$ \\
Stable & 40 & 15.0 & $(3)$ \\
Increase and stabilize & 18 & 6.8 & $(4)$ \\
Miscellaneous & 15 & 5.6 & \\
Stable and decrease & 14 & 5.3 & $(5)$ \\
Total & 266 & 100.0 & \\
\hline
\end{tabular}

- is stable,

- increases and stabilizes,

- is stable and decreases,

- increases and decreases.

Necks that continuously decrease in size represent about $26 \%$ of the necks. They link a small particle, which is dissolving, to a bigger one, which is slightly growing; the observed mechanism can be assimilated to Ostwald ripening though particles are connected. The rate of dissolution of the small particle and the corresponding neck, i.e. the slope of curve (1) in Fig. 10a, depends on the initial size of the neck at $t_{0}$. Most of the data for decreasing necks fall in the scatter-band represented in Fig. 10a; an average curve was computed from all the curves obeying trend (1). The higher the initial surface of the neck, the lower the rate of neck decrease; thus large necks are close to the upper bound of the scatter-band. Conversely, a large difference in sizes between the two particles on both sides of the neck favours a rapid kinetics since the difference in globule curvatures is the driving force for mass transfer. Neck no. 39 was selected for detailed observation as it is a good representative of this trend; by the time it is detected, i.e. near $t_{0}=1500 \mathrm{~s}$, it connects a small particle, numbered (1) in Fig. 11b, to a larger one, numbered (2). Observation of the neck is not easy as particle (1) has six bonds that are visible in Fig. 11a. Thus only some particles, numbered from 1 to 5 in Fig. 11a, were chosen for observation in Fig. 11b. Fig. 11b-d shows the evolution of this cluster of particles with holding time. The progressive dissolution of the small solid globule (1) is clearly seen but the coarsening of its neighbours is not large enough to be visible to the naked eye. The flux of matter leaving particle (1) is not oriented towards particle (2) only; contacts with particles (3) and (4) are maintained until complete disappearance of particle (1) and these are only part of the real number of contacts of particle (1). From $1560 \mathrm{~s}$ to $3960 \mathrm{~s}$ as particle (1) decreases constantly in size, the other particles slightly spheroidize and particles (2) and (5) grow.

Necks that continuously enlarge are as numerous as the continuously decreasing necks of trend (1); they belong to trend (2) plotted in Fig. 10a. The initially small necks grow faster than the large ones so that their curves are close to the upper bound of the scatter-band. The average neck surface for trend (2) depends on time to the power $1 / n$ with $n=2.4$, i.e. the neck radius varies as $t^{1 / 4.8}$. Neck no. 134 has an evolution that is close to the average of trend (2), being thus well representative of this trend. It links two particles of similar size, which are numbered (1) and (2) in Fig. 12. Note that while the neck no. 134 is increasing, necks between (2) and (3) or (2) and (4) remain stable. A flux of matter from particles (1) and (2) gradually fills the neck in between reducing its curvature. While the neck diameter rapidly enlarges, the particle radii decrease slowly, which is typical of coalescence. The shape factor of solid globules increases from 1560 to $2760 \mathrm{~s}$ especially for particle (1), which has agglomerated with a smaller particle. For 

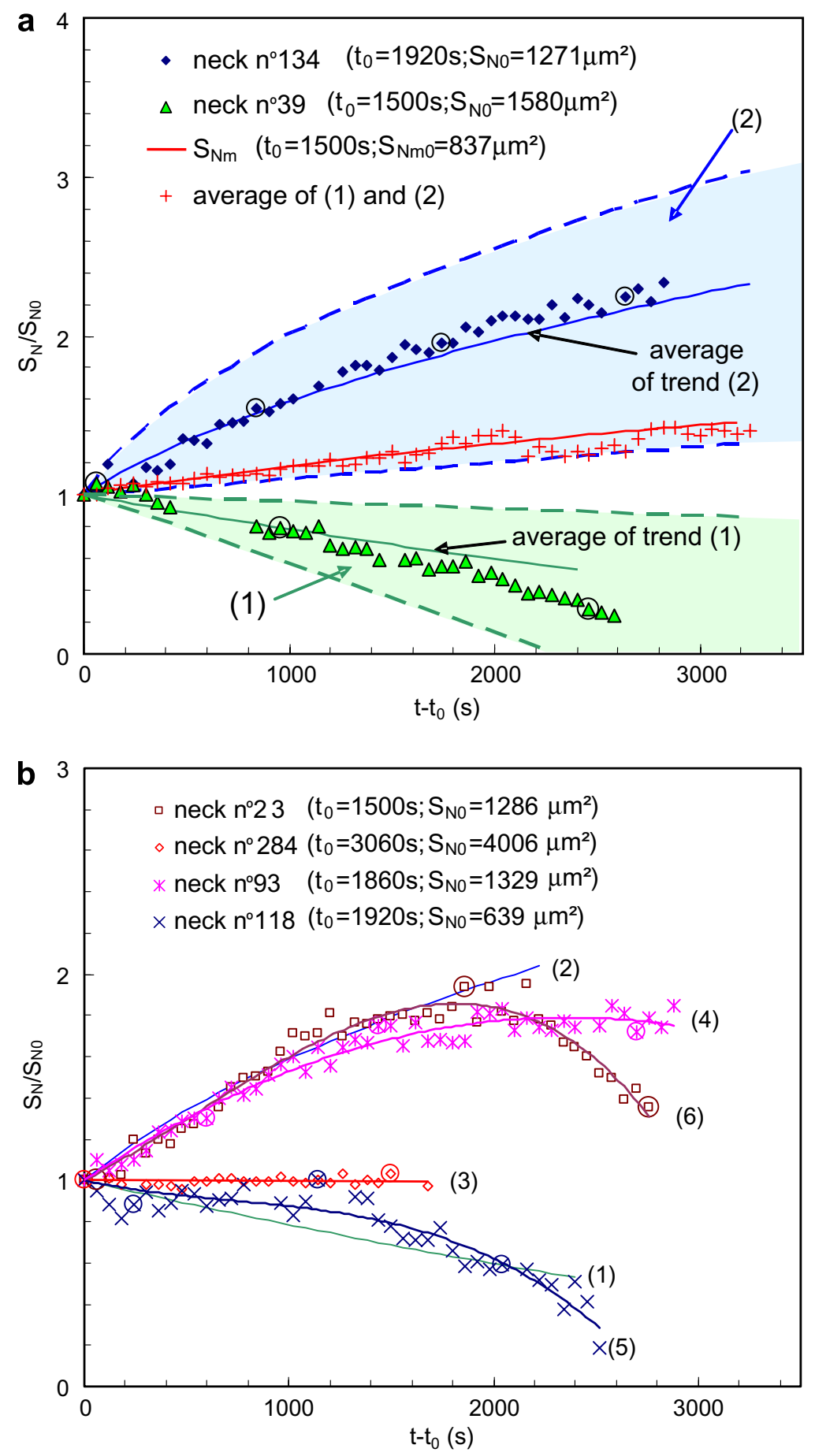

Fig. 10. Size variation for (a) continuously increasing (2) or decreasing (1) necks and for (b) necks that show more complex behaviour under the influence of the neighbourhood.

longer holding times, coalescence between (1) and (2) prevents these particles from further spheroidization.

$15 \%$ of the necks have a constant size during the time of their analysis. This trend, numbered (3) in Fig. 10b, is well illustrated by neck no. 284 between particles (1) and (2) in Fig. 13; the sizes of the necks and particles are stable during the time of their observation, i.e. during about $1600 \mathrm{~s}$. However, before its detection at $t_{0}=3060 \mathrm{~s}$, this neck was growing by coalescence from at least $1060 \mathrm{~s}$ to 2460 s. Afterwards it maintains the same size with a ratio of neck to particle radius of about 0.68 . The small curvature at the neck root and the large ratio of neck to particle radius could correspond to a stable configuration [27] where the flux of matter from the particles to the neck area is not favoured any more. However, it is more difficult to explain why some necks that are small compared to the 


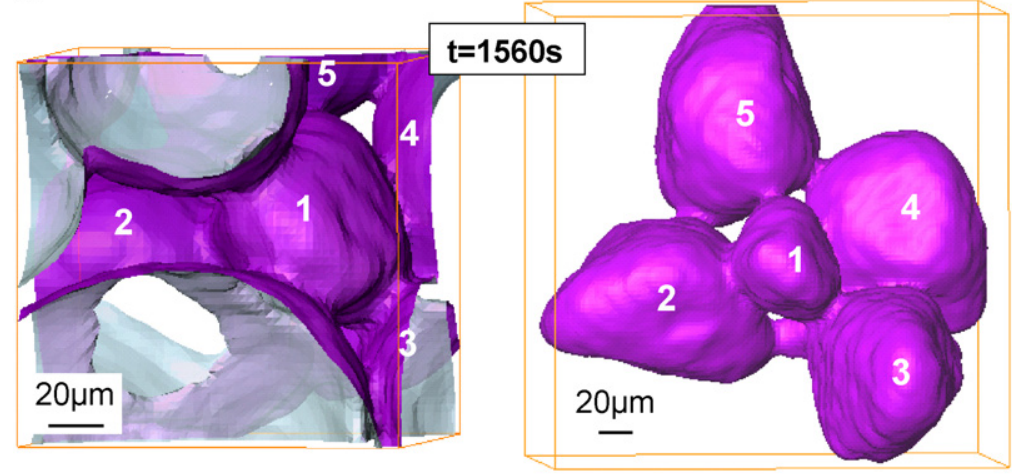

C

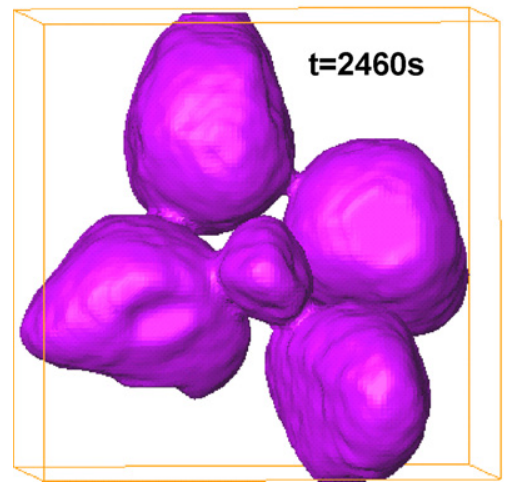

d

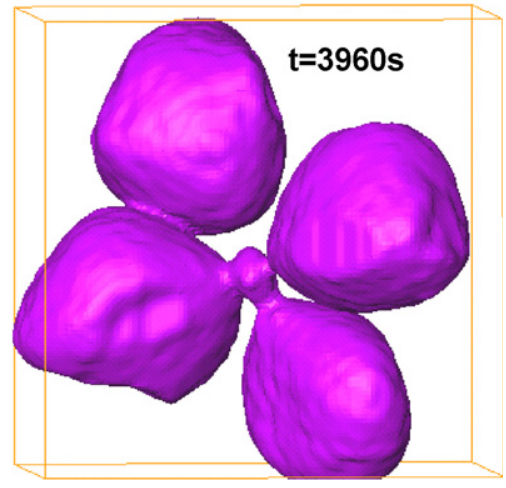

Fig. 11. Observations of neck no. 39 representative of trend (1).

particles they link, e.g. the neck between particles (2) and (3) in Fig. 13, were also observed to remain stable; this is probably due to the influence of other solid particles in the neighbourhood.

About $7 \%$ of the necks grow until they reach a stable size according to trend (4) (Fig. 10b). For example, neck no. 93, which links particles (1) and (2) in Fig. 14, enlarges from $t_{0}$ to $3300 \mathrm{~s}$ and then it remains stable when the ratio of neck to average particle radius has reached about 0.5 . We believe that in some cases trend (4) is similar to trend (3) but here, the timescale of the analysis was large enough for the initial coalescence mechanism to be observed in addition to the stable part. As mentioned before, coalescence could stop or slow down when the ratio of neck radius to particle radius is large. However, some necks were observed to grow from almost zero to stability at a neck to particle size ratio less than 0.3. Explanation of this phenomenon would require knowledge of how the particles that are adjacent to the pair of particles implied in the neck are evolving. Coalescence and "Ostwald ripening" are two coarsening mechanisms in competition and local predominance of one mechanism depends on the neighbourhood. Though the neck between (1) and (2) is stable until $4560 \mathrm{~s}$, particle (1) is dissolving, which might lead to a decrease in neck size at longer holding times.

Indeed, the 5\% of the necks, which compose trend (5), stay constant for a while then decrease until final disap- pearance of one of the particles adjacent to the neck. For example neck no. 118 in Fig. 15 links the small particle (1) to particle (2), which is almost twice as big. The neck has an initial size that corresponds to a rather small neck to particle ratio, i.e. 0.25 and 0.45 for particles (2) and (1), respectively; however, the neck remains at a stable size for at least $1000 \mathrm{~s}$. For the sake of visibility, three out of the four direct neighbours of particle (1) were represented in Fig. 15. Particles (1) and (4), which are of the same size, are strongly connected and their diameters decrease at a similar rate since they are being dissolved to the benefit of the surrounding bigger particles. Obviously, dissolution of particle (1) is responsible for the decrease of the neck between (1) and (2). Again, this trend would be impossible to explain without taking into account the neighbourhood.

$15 \%$ of the necks grow first and then decrease in size according to trend (6) in Fig. 10b. For example, neck no. 23 shows a rapid increase in size when the two particles, i.e. (1) and (2) in Fig. 16, bonded through this neck, begin to coalesce. From $2760 \mathrm{~s}$ to $3360 \mathrm{~s}$, it grows more slowly, maybe because the ratio of neck to particle radius has become rather high, i.e. about 0.6 . The two particles have initially similar sizes but particle (1) is bonded to a bigger particle numbered (3) while particle (2) is bonded to a smaller particle numbered (4). In each case, the smaller particle dissolves at the benefit of the larger one. Dissolution of particle (1) means a decrease of the neck diameter between 

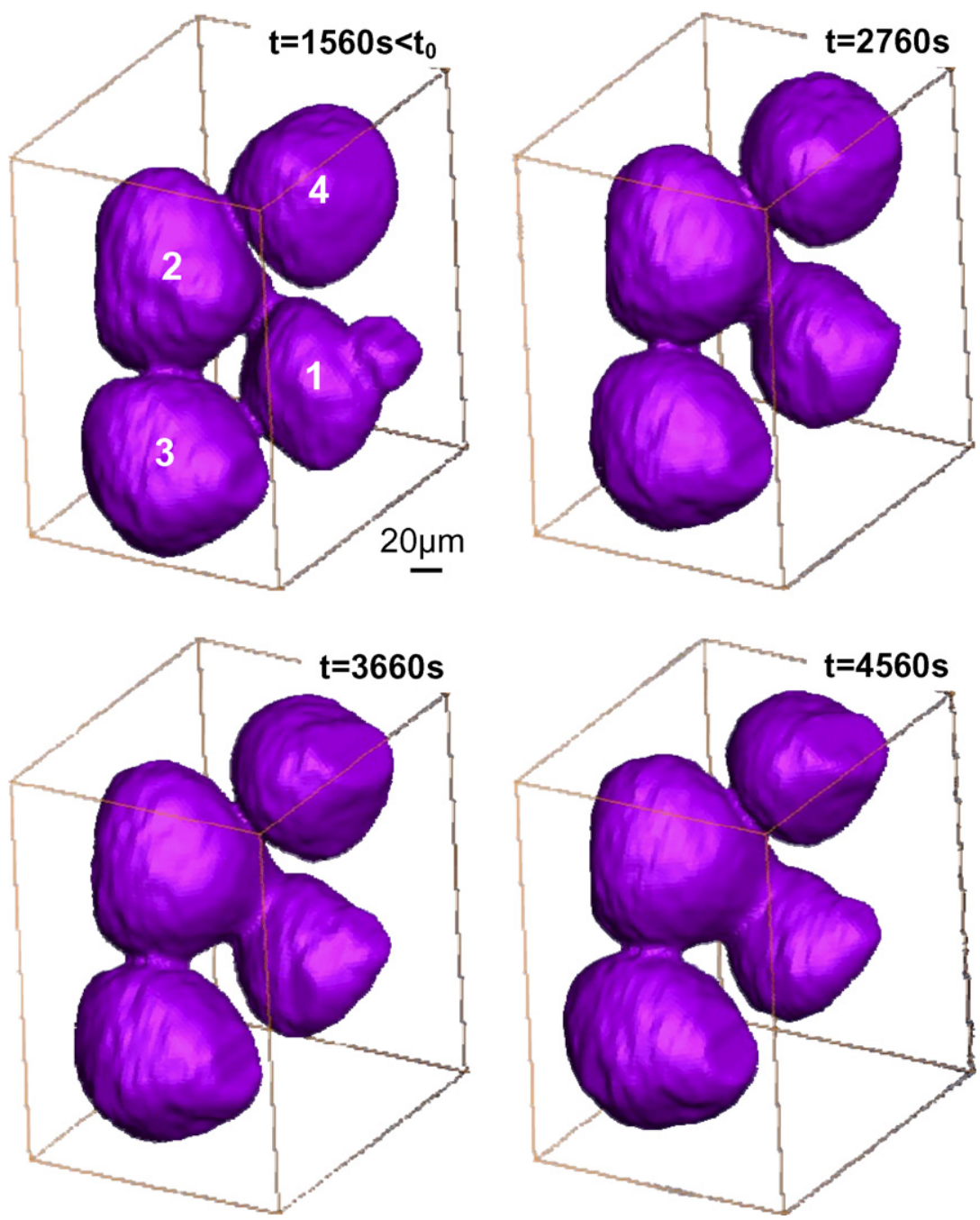

Fig. 12. Observations of neck no. 134 representative of trend (2).

(1) and (2) with holding longer than $3360 \mathrm{~s}$. Note that some necks did not grow that big before the tendency is reversed, that is to say that they begin to decrease soon after the coalescence probably because a more favourable flux drives the matter away from the neck.

Some necks were not classified. They are reported as miscellaneous in Table 1 because their sizes do not follow one of the above-mentioned trends and they are not numerous enough to define a uniquely different trend.

\section{Discussion}

The experimental particle density does not show the standard $1 / t$ dependency for Ostwald ripening, which proves that the LSW model is unable to account for the complexity of the microstructural mechanisms that are taking place in the system considered here. Two coarsening mechanisms were observed that both act upon the number of solid particles. In the dissolution-reprecipitation mechanism, small particles are absorbed by larger particles in an Ostwald ripening-like mechanism where particles are not isolated but connected to each other. A higher concentration of solute around the small particle with high curvature creates a lower melting point and favours dissolution of the small particle. The solute is then deposited on larger particles in the neighbourhood of the small particle. Coalescence between two particles of similar size could also decrease the number of particles when the neck between them has grown to such an extent that the original particles cannot be distinguished and segmented any more. We did not observe complete coalescence in this study but neck growth could become slower when the neck has increased to a large fraction of the particle diameter. The ratio of neck to particle radius was generally well below the value of 0.7, which Courtney chose for complete coalescence; the approximate percentage of necks that grow up to this value was estimated to be less than $3 \%$. Difficulty to reach complete coalescence might be ascribed at least partially to the neighbourhood: there is a strong possibility that neck growth is stopped or even reversed before coalescence is finished. As a result, the time dependency (also in $1 / t$ ) developed by Courtney for times larger than the time 

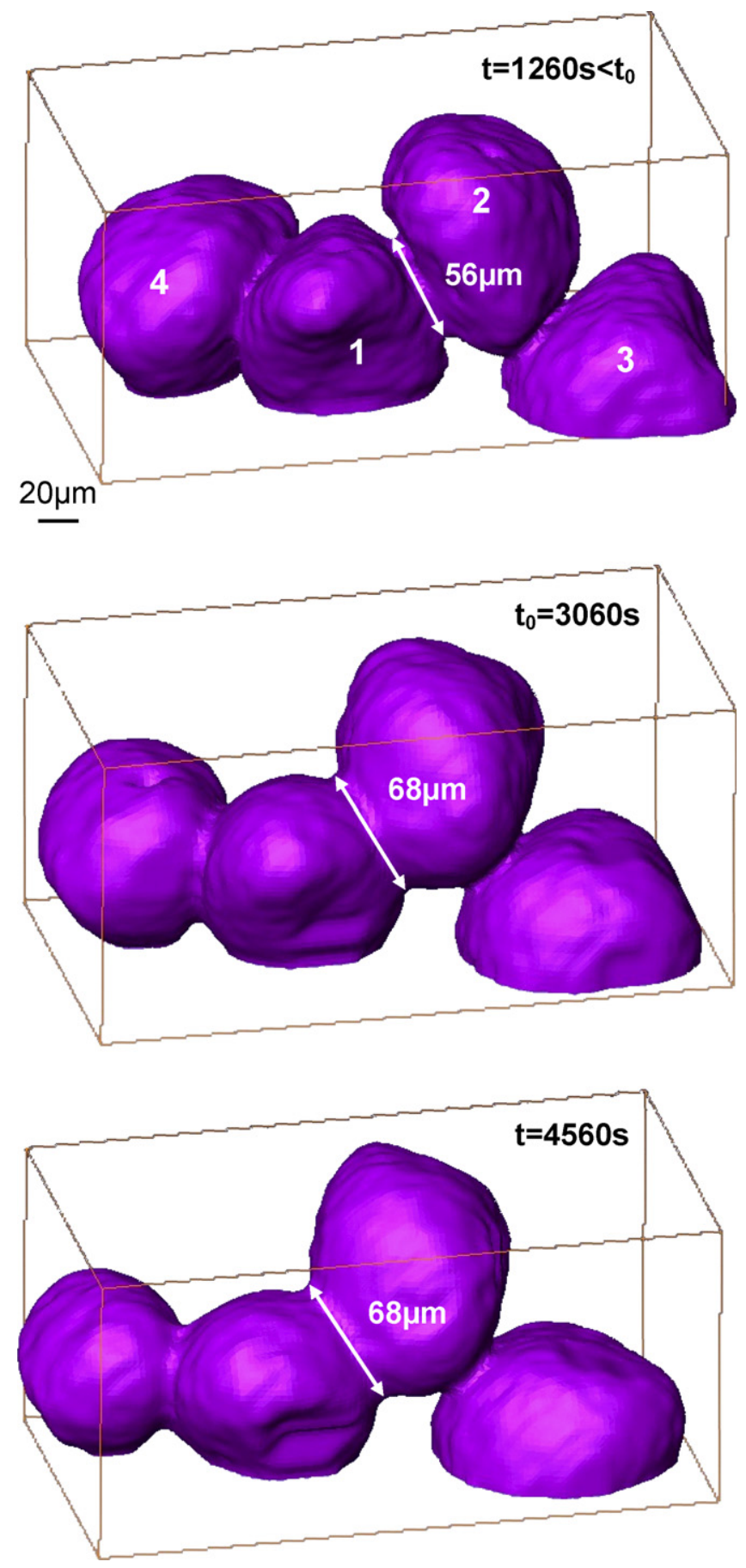

Fig. 13. Observations of neck no. 284 representative of trend (3).

required to fuse some of the particles together would not fit our results in a better way; coalescence has almost no effect on the number of solid particles.

To minimize surface energy, the ratio of surface to volume of solid particles has to decrease. Thus, spheroidization of the solid phase occurs because spheres have a lower surface-to-volume ratio than all other shapes. However, this shape evolution is constrained by the connectivity of the system so that the mean shape factor does not exceed 0.845. Each time two particles of different sizes merge because of dissolution-reprecipitation, they form a solid particle with a shape factor smaller than the average value. For example, in Fig. 16, as soon as particles (1) and (3) on one hand and particles (2) and (4) on the other hand are no longer separable by segmentation, the so-formed agglomerates have shape factors around 0.75 that rise to 0.845 with longer holding times. Ferrante and de Freitas [28] also observed this slow-down in shape change that they attributed to hard impingement of solid particles, i.e. coalescence.

The average coordination number, $z$, is close to the number of contacts that could be made between spheres in random loose packing, i.e. around 6.4, although the particle-particle contacts in the semi-solid are not points but necks with a finite size. The slight decrease of $z$ with an increase in holding time could correspond to the slow shape evolution; the coordination number decreases as the particle tends to develop a more spherical shape because spheres cannot make as many contacts as ellipsoids for example. Dissolution of small particles could also influence the $z$ value. On one hand, dissolution of a solid particle implies loss of the necks that this particle has made with its neighbours (see for instance the loss of the neck between particles (1) and (5) in Fig. 11). On the other hand, shrinking of a small particle tends to bring its growing neighbours closer to each other allowing new contacts to be created by coalescence. "Ostwald ripening" and coalescence are not expected to have a large influence on the coordination number since the effects of the two mechanisms tend to annihilate each other. Finally, in some cases, adjacent growing necks were observed to merge diminishing the number of necks for the particle under consideration.

Assuming that the variation of the average volume of particle is linear with time, i.e. the average equivalent radius obeys a $t^{1 / 3}$ power law, the two slopes in Fig. 6 could correspond to the transient and permanent regimes, respectively. Actually, as the size distribution in Fig. 7 is not perfectly independent of time, it is possible that the permanent regime hypothesis, which is a prerequisite for LSW validity, is not verified. Snyder et al. [29] noticed that transient regime could be very long and that steady state may not be reached for certain systems during the time of the experiment; this results in a rate constant $K$ larger than the "theoretical" LSW value.

The evolution of $S_{\mathrm{v}}$ with time does not sustain the above-mentioned hypothesis (Fig. 8). From the time where $g_{\mathrm{s}}$ reached its equilibrium value to the end of the isothermal holding experiment, a unique $t^{-1 / n}$ power law with $n$ equal to 7.5 could fit the evolution of $S_{\mathrm{v}}$. If in situ experiments are compared to post-quench experiments on the same alloy [19], the quenched specimen appears to have a higher $S_{\mathrm{v}}$ value than the semi-solid specimen and the discrepancy between the two curves in Fig. 8 decreases with increasing holding time. During quenching, the primary Al-rich phase deposits on the existing solid globules upon cooling. Thus the particle average size is overestimated as well as the solid 

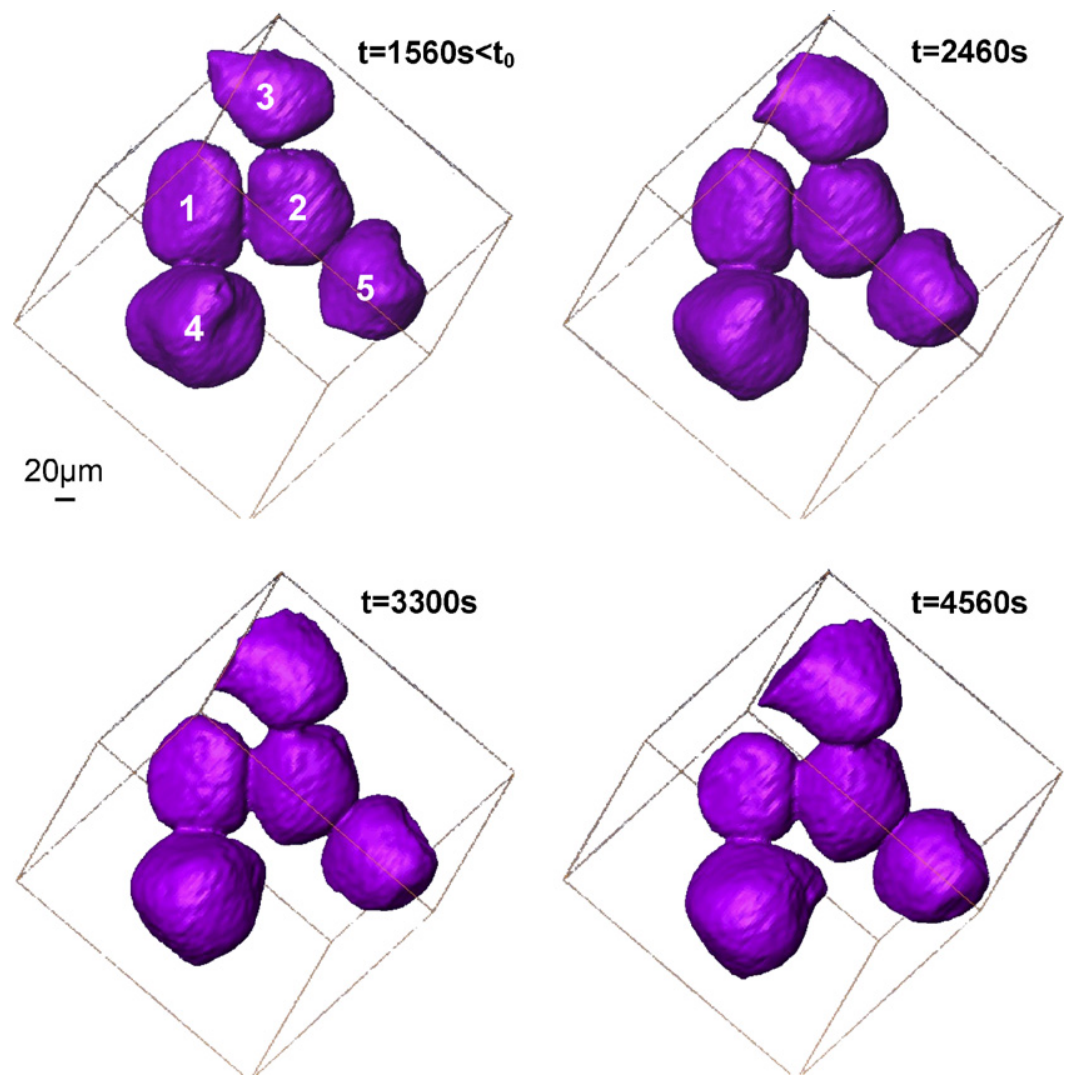

Fig. 14. Observations of neck no. 93 representative of trend (4).

fraction and the solid-liquid surface area as compared to the semi-solid state. $S_{\mathrm{v}}$ of the quenched specimen has a time coefficient higher than the $1 / 7.5$ coefficient of the in situ experiment. A $1 / 3$ coefficient was initially found [19] but a smaller coefficient equal to $1 / 4.8$ gives a better fit in Fig. 8. In addition, assuming an asymptotical behav-
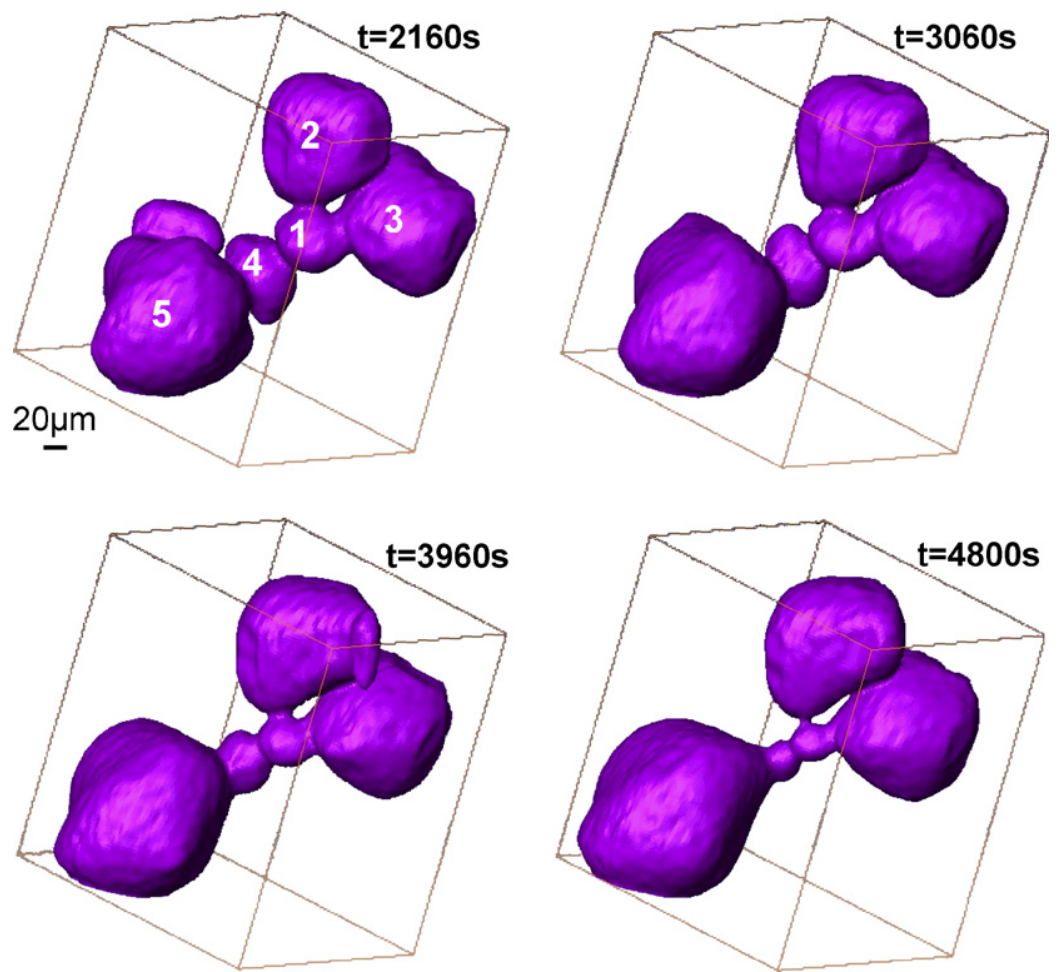

Fig. 15. Observations of neck no. 118 representative of trend (5). 

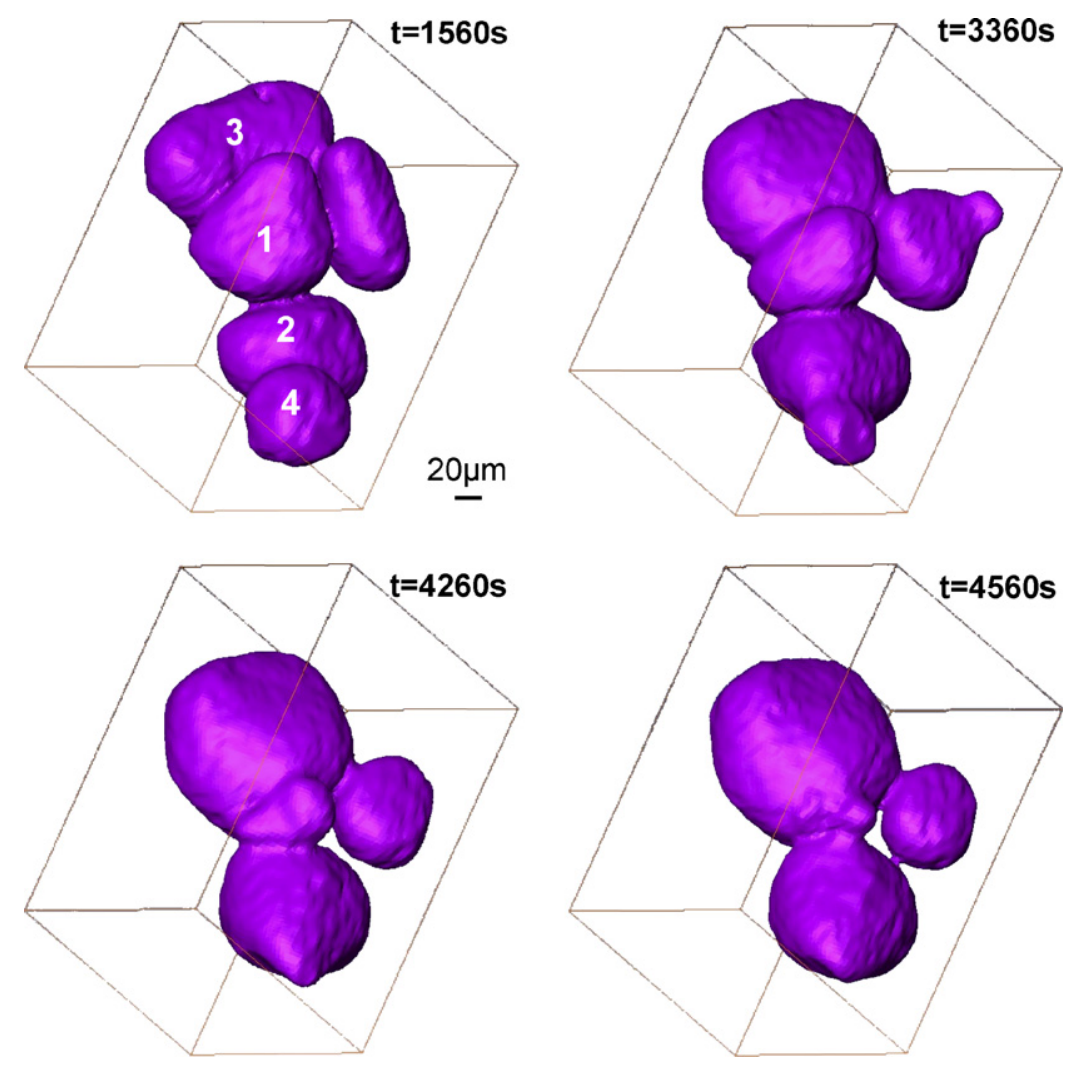

Fig. 16. Observations of neck no. 23 representative of trend (6).

iour at long holding times, a 1/6 time exponent could also fit the data; this proves that obtaining an accurate value of the time coefficient requires more data points. Most of the time, a 1/3-power law is assumed to hold even at a high volume fraction of solid although there are not enough results to sustain this assumption. Unlike post-quench measurements, in situ tomography gives measurements that are representative of the semi-solid structure and that are numerous enough to draw satisfactory fits.

The average surface area of necks, $S_{N m}$ in Fig. 9, increases with time to the power $1 / 2.9$, which is close to the values proposed by Courtney [11] for the increase in neck radius by coalescence in liquid-phase sintering. Actually, the relative surface of necks that grow by "pure" coalescence, i.e. with a size evolution that pertains to trend (2), has a similar time exponent of about $1 / 2.4$ as shown by the average curve plotted in Fig. 10a. When the neck has a large size at $t_{0}$, i.e. its relative surface is close to the lower bound of the scatter band in Fig. 10a, its time evolution cannot be analysed with the same exponent. It is possible that once the neck has grown to a large size compared to the particle radius, it becomes stable or grows at a lower rate maybe under the influence of the two particles that it links and that could grow by "Ostwald ripening" [13]. As reported in Table 1, trends (1) and (2), i.e. "Ostwald ripening" and coalescence, occur with the same weight. Consequently, an average curve was calculated directly from the average of both trends. The resulting points represented by cross symbols in Fig. 10a are well fitted by $S_{N m}$. The average size of necks tends to increase with time due to both coalescence and dissolution of small necks between particles of dissimilar sizes. Nevertheless, although continuously decreasing and increasing neck sizes seem sufficient to explain the evolution of average values, we observed more complex neck evolutions that cannot be understood if the influence of the neighbourhood is ignored.

Analysis of the microstructural parameters measured from 3D data obtained with in situ tomography shows that the solid volume fraction of 0.68 is high enough for the structure to consist of solid interconnected globules that cannot be considered as disperse spheres like in the LSW theory. The number of contacts per particle ranges from 1 to 13 so that there is no free particle and the existence of necks between particles implies that coalescence can occur when two particles in contact are of similar size and that the neighbourhood does not prevent coalescence. Tzimas and Zavaliangos [30] note that the coalescence frequency being proportional to the average number of necks per particle according to Kaysser et al. [26], the coalescence mechanism should be significant for high solid fractions. Indeed Courtney's model for coalescence predicts time exponents that are closer to our experimental results than the LSW model.

The study of local evolution of the microstructure between $1500 \mathrm{~s}$ and $4800 \mathrm{~s}$ of holding time at $555^{\circ} \mathrm{C}$ allows understanding of the unconventional time dependency of 
the solid-liquid interface area and to determine the relative importance of coalescence compared with ripening. From our results, coalescence was proved as important as dissolution-reprecipitation. Both can exist as pure mechanisms, i.e. trends (1) and (2) in Fig. 10a. By pure mechanism we mean a coarsening mechanism that is not visibly affected by the neighbourhood, i.e. the neck will continuously grow, shrink, or remain stable. In many cases, ripening and coalescence do not act independently because on a given local feature of the microstructure, such as a neck between two solid particles, several mechanisms were observed. In Fig. 16 representative of trend (6), "Ostwald ripening" between particles (1) and (3), clearly affects coalescence between particles (1) and (2); thus the kinetics of coalescence depends on the coarsening mechanisms that are active in the neck vicinity. The neighbourhood influence is obvious in trends (5) and (6) and it possibly affects trends (3) and (4) under certain conditions. As mentioned, stability, i.e. trend (3), could result directly from pure coalescence as two particles cannot fuse at the same rate indefinitely and larger necks were indeed observed to grow at a lower rate in Fig. 10a; then, it would be considered as a pure mechanism. Trend (3) could result also from the influence of the neighbourhood, especially when it occurs at a small neck-to-particle ratio. The neck between two strongly coalesced particles is likely to remain stable if the particle's size is greater than the average, which, according to the LSW model corresponds to the critical size below which a particle could be dissolved. For example, particle (2) in Fig. 13 has an equivalent radius well above the average, i.e. of about $54 \mu \mathrm{m}$, and the neck was indeed observed to remain stable over a long time period. On the contrary, particles connected by neck no. 23 in Fig. 16 have equivalent radii less than or equal to $47 \mu \mathrm{m}$, i.e. below the average or critical size, so that the neck size is likely to be under the influence of the neighbourhood instead of maintaining a stable size.

"Ostwald ripening" and coalescence were proved to occur simultaneously. As a result, they both contribute to coarsening of the microstructure but not necessarily in the same way. "Ostwald ripening" increases the average equivalent radius of the solid globules whereas coalescence would rather slightly decrease it to an extent that may be not visible. Globally, the average equivalent radius of the structure should have an evolution with time that lies between the $t^{1 / 3}$ power law for ripening and the almost constant value for coalescence (as long as coalesced particles could be segmented). Furthermore, as noted by Kim and Yoon [8], the particle surface that is taken to create a grain boundary or a neck is shielded from the dissolution-reprecipitation process. Consequently, the increase of the average particle radius due to ripening could be smaller than if dissolution-reprecipitation was the single coarsening mechanism. Regarding the evolution of $S_{\mathrm{v}}$, coalescence mainly decreases the solid surface area near the neck roots (see for instance the differences between $1560 \mathrm{~s}$ and $4560 \mathrm{~s}$ in Fig. 12) while ripening influences the particle diameters.
In the original LSW theory, the solid surface would correspond to the particle density multiplied by the surface of a solid particle with a radius equal to the average equivalent radius. This computation gives a value for $S_{\mathrm{v}}$ that is about $25 \%$ higher than the measured value and that evolves at a much lower rate. The surface of isolated spheres overestimates $S_{\mathrm{v}}$ because it neglects the surface area occupied by the necks and its evolution with holding time. If we consider pure coarsening mechanisms, $S_{\mathrm{v}}$ is expected to grow as $t^{-1 / 6}$ or $t^{-1 / 5}$ for coalescence and as $t^{-1 / 3}$ for Ostwald ripening. Thus it is difficult to explain how, in a semi-solid microstructure where both mechanisms are observed, the measured $S_{\mathrm{v}}$ varies with time with an even smaller coefficient, i.e. $1 / 7.5$, than for pure mechanisms. A possible explanation would be that, since these mechanisms do interfere, as shown by the necks evolution, they may delay each other.

Since almost half the observed necks visibly evolve under the influence of the neighbourhood, a model that would consider diffusion between two particles only would have limited use. Eggleston [31] suggested using a phase field model as a basis for simulation of hundreds of particles undergoing "Ostwald ripening". However, as there is no predominance of one coarsening mechanism during the timescale when we observed the local evolution of necks, neither a model developed for pure coalescence nor a model developed for pure dissolution-reprecipitation would be able to account for the actual complexity of the system.

\section{Conclusions}

The real-time microstructural evolution of an $\mathrm{Al}-$ 15.8 wt. $\% \mathrm{Cu}$ alloy during partial remelting was investigated with in situ fast X-ray tomography. Isothermal holding was performed during $80 \mathrm{~min}$ at a temperature of $555^{\circ} \mathrm{C}$, which resulted in a solid volume fraction of 0.68 . The starting microstructure of the analysed sample was globular. A tomographic scan was acquired every minute. In addition to the conventional characteristic microstructural parameters, the local evolution of microstructural features in the semi-solid state was analyzed and illustrated with 3D images. The main conclusions are the following:

1. The average values of the microstructural parameters, such as the particle density and size, and the area of the solid-liquid interface, $S_{v}$, do not follow the standard power laws associated with Ostwald ripening.

2. The time variation of $S_{\mathrm{v}}$ is shown to obey a power law with a time exponent of about $1 / 7$. This is close to Courtney's model for coalescence in liquid-phase sintering.

3. Two coarsening mechanisms, which have equivalent contribution, are shown to act upon the microstructural evolution of the solid phase during isothermal holding: Dissolution of a small particle to the benefit of one or several bigger particles by an Ostwald-like mechanism 
is observed as well as the growth of necks between solid globules due to coalescence of particles with comparable sizes.

4. The variation with time of average and local microstructural parameters can be interpreted by taking into account the coexistence of two competing coarsening mechanisms that may have opposite influence. For example, "Ostwald ripening" increases the average equivalent radius of a solid globule whereas coalescence slightly decreases it as long as the two coalesced particles can be separated.

5. Locally, the semi-solid microstructure evolves by a combination of the two "pure" coarsening mechanisms. The evolution of necks with mixed behaviour, e.g. necks that increase in size, remain stable for a while, and eventually decrease, could only be explained by the influence of the neighbourhood as confirmed by $3 \mathrm{D}$ observations.

6. In situ fast X-ray microtomography is proved to be a powerful tool to provide data that are representative of the semi-solid state and to observe in real-time the mechanisms that act on the microstructure. Further experiments are planned to study the influence of the solid fraction on the microstructural evolution during isothermal holding.

\section{Acknowledgements}

Part of this work was carried out in the framework of the Project ANR-05-BLAN-0286-01 "TOMOSOLIDAL" supported by the Agence Nationale de la Recherche which is gratefully acknowledged. The authors thank Øyvind Nielsen from SINTEF for providing the material of this investigation. The authors from INPG are also grateful to ESRF for providing beam time on the ID15 beam line and to all staff members of the line for their help during the experiments.

\section{References}

[1] Wilson PW, Kattamis TZ, Shiohara Y. J Mater Sci 1988;23:2882.

[2] Kattamis TZ, Coughlin JC, Flemings MC. In: Abbaschian R, Brody $\mathrm{H}$, Mortensen A, editors. Proceedings of the Merton C. Flemings symposium on solidification \& materials processing. TMS; 2001. p. $35-42$.

[3] Kattamis TZ, Coughlin JC, Flemings MC. Trans Metall Soc AIME 1967;239:1504.

[4] Duncan AJ, Han Q, Viswanathan S. Metall Mater Trans B 1999;30:745.

[5] Poirier DR, Ganesan S, Andrews M, Ocansey P. Mater Sci Eng A 1991;148:289.

[6] Chen M, Kattamis TZ. Mater Sci Eng A 1998;247:239.

[7] Mortensen A. Met Trans A 1991;22:569.

[8] Kim SS, Yoon DN. Acta Metall 1983;31:1151.

[9] Kailasam SK, Glicksman ME, Mani SS, Fradkov VE. Metall Mater Trans A 1999;30:1541.

[10] Zukas EG, Rogers PSZ, Rogers RS. Z Metall 1976;67:591.

[11] Courtney TH. Met Trans A 1977;8:671.

[12] Courtney TH. Met Trans A 1977;8:679.

[13] Courtney TH. Met Trans A 1977;8:685.

[14] Bender W, Ratke L. Acta Mater 1998;46:1125.

[15] Voorhees PW, Schaefer RJ. Acta Metall 1987;35:327.

[16] Mendoza R, Alkemper J, Voorhees PW. Metall Mater Trans A 2003;34:481

[17] Rowenhorst DJ, Kuang JP, Thornton K, Voorhees PW. Acta Mater 2006;54:2027.

[18] Pompe O, Rettenmayr M. J Cryst Growth 1998;192:300.

[19] Salvo L, Suéry M, Josserond C, Cloetens P, Nielsen Ø. In: Tsutsui Y, Kiuchi M, Ichikawa K. editors. Proceedings of the 7th S2P conference on advanced semi-solid processing of alloys and composite, Tsukuba; September 25-27 2002. p. 403.

[20] Ludwig O, DiMichiel M, Falus P, Salvo L, Suéry M. In: Proceedings of the 8th S2P conference on semi-solid processing of alloys and composites, Limassol, Cyprus, 21-23 September 2004, published as CD-ROM by NADCA, USA.

[21] Ludwig O, DiMichiel M, Salvo L, Suéry M, Falus P. Metall Mater Trans A 2005;36:1515.

[22] Ganesan S, Poirier DR. Metall Trans A 1987;18:721.

[23] M. Braccini, Ph.D. thesis, Institut National Polytechnique de Grenoble, 2000.

[24] Sarian S, Weart HW. J Appl Phys 1966;37:1675.

[25] Lifshitz IM, Slyozov VV. J Phys Chem Solids 1961;19:35.

[26] Kaysser WA, Takajo S, Petzow G. Acta Metal 1984;32: 115.

[27] German RM. Sintering theory and practice. Wileys \& Sons; 1996. 225-312.

[28] Ferrante M, de Freitas E. Mater Sci Eng A 1999;271:172.

[29] Snyder VA, Alkemper J, Voorhees PW. Acta Mater 2001;49: 699.

[30] Tzimas E, Zavaliangos A. Mater Sci Eng A 2000;289:228.

[31] J. Eggleston, Ph.D. thesis, Northwestern University, 2001. 